\title{
Evaluation of the effect of chiropractic manipulative treatment on oxidative stress in sacroiliac joint dysfunction
}

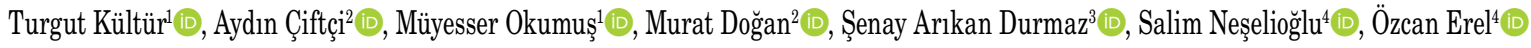 \\ ${ }^{1}$ Department of Physical Therapy and Rehabilitation, Kırıkale University Faculty of Medicine, Kırıkkale, Turkey \\ ${ }^{2}$ Department of Internal Medicine, Kırıkkale University Faculty of Medicine, Kırıkkale, Turkey \\ ${ }^{3}$ Department of Internal Medicine, Division of Endocrinology and Metabolic Diseases, Kırkkkale University Faculty of Medicine, Kırıkkale, Turkey \\ ${ }^{4}$ Department of Medical Biochemistry, Ylldrrm Beyazit University Faculty of Medicine, Ankara, Turkey
}

Received: July 03, 2018 Accepted: December 13, 2018 Published online: May 18, 2020

\begin{abstract}
Objectives: This study aims to investigate the effect of chiropractic manipulative treatment on sacroiliac joint dysfunction (SIJD) and its relationship to oxidative stress (OXS) parameters.

Patients and methods: Thirty-three patients diagnosed with SIJD (20 males, 13 females; mean age $36.3 \pm 9.7$ years; range, 18 to 60 years) and 30 healthy volunteers ( 20 males, 10 females; mean age $36.4 \pm 12.2$ years; range, 20 to 57 years) were included in this cross-sectional, case-control study conducted between February 2017 and September 2017. Manipulation was applied to the patients once a week for a duration of four weeks. The patients were evaluated at pre-treatment and one month after treatment with visual analog scale, SIJD test, and total thiol, native thiol, disulphide, and ischemia-modified albumin (IMA) as OXS indicators.

Results: Prior to treatment, we demonstrated that serum native thiol $(\mu \mathrm{mol} / \mathrm{L})$ and total thiol $(\mu \mathrm{mol} / \mathrm{L})$ levels in the patient group were lower compared to control subjects $(\mathrm{p}=0.03$ and $\mathrm{p}=0.02$, respectively). Serum IMA levels were higher in the patient group ( $\mathrm{p}=0.01$ ). There was no change in OXS parameters after manipulative treatment in the patient group.

Conclusion: Manipulation is useful in SIJD. Thiol/disulphide homeostasis and serum IMA levels may be used to measure the OXS in patients with SIJD.
\end{abstract}

Keywords: Chronic pain, ischemia-modified albumin, manipulative treatment, sacroiliac joint dysfunction, thiol/disulphide homeostasis.

The excessive increase of free oxygen radicals produced as a defense mechanism may result in oxidative stress (OXS) damaging the body. Cancer, heart disease, diabetes, vascular disease, and musculoskeletal diseases such as osteoarthritis, rheumatoid arthritis (RA), and fibromyalgia (FM) may cause OXS. Moreover, OXS may contribute to the development or worsening of these diseases. ${ }^{[1-4]}$

Thiol-disulphide balance is a new method for determining OXS. ${ }^{[5]}$ The thiols can form disulphide bonds by entering into the oxidation reaction via oxidants. Increased OXS can lead to the reversible formation of mixed disulphides between thiols and protein thiol groups. The resulting disulphide bonds can be reduced back to thiol groups and thiol-disulphide homeostasis can be protected. Thus, the thiol/disulphide ratio has been shown to play a critical role in detoxification, antioxidant protection, signal transduction, regulation of enzymatic activity, apoptosis, and cellular signaling. ${ }^{[5-7]}$

Human serum ischemia-modified albumin (IMA) has been studied and regarded as a sensitive biomarker for diagnosis of many OXS-related clinical conditions. Human serum albumin converted to IMA when the albumin $\mathrm{N}$ terminus changed due to OXS or ischemia. In addition to cardiovascular events, serum IMA levels were found to be significantly elevated in painful chronic musculoskeletal conditions such as FM, ankylosing spondylitis (AS), and RA. ${ }^{[8-10]}$ Sacroiliac (SI) pain due to impaired joint function

Corresponding author: Turgut Kültür, MD. Kırıkkale Üniversitesi Tıp Fakültesi Fiziksel Tıp ve Rehabilitasyon Anabilim Dalı, 71450 Yahşihan, Kırıkkale, Türkiye. 
in SI dysfunction may be the cause of chronic low back pain. ${ }^{[1]}$ Oxidative stress increases production of cytokines such as interleukin-1beta (IL-1 $\beta$ ), tumor necrosis factor-alpha, and IL-6. Moreover, in OXS, nuclear factor kappa B (NF-kB) activates the genes of these cytokines by causing up-regulation of toll-like receptor 4 (TLR-4). Nuclear factor- $\kappa B$ and TLR-4 inhibit sirtuin 1 (SIRT1) gene. SIRT1 has antiinflammatory and anti-oxidative effects.

Oxidative stress decreases SIRT1 activity, leading to increased NF- $\kappa B$ activity. Nuclear factor- $\kappa B$ causes inflammatory responses. Increased cytokines and inflammation may lead to damage in lipids, protein and deoxyribonucleic acid (DNA) and have devastating effects on cellular function. Chronic inflammatory tissue damage and elevated levels of cytokines play an important role in the pathogenesis of low back pain. In addition, OXS may contribute to the development of chronic pain by enhancing neuronal activity, central and peripheral sensitivity, and affecting the interneurons of the spinal cord dorsal horn. In pain, increased neuronal metabolism and enhanced use of the metabolic substrates can produce OXS. Inflammation, pain, and OXS stress can increase the need for oxygen. Oxidative stress and pain may be exacerbated by increased ischemia. The result is a vicious cycle of pain, inflammation, and OXS that trigger each other. Thus, pain and OXS may be chronic. ${ }^{[1-4,11-13]}$ In this context, sacroiliac joint dysfunction (SIJD) may cause OXS or may implicate oxidant, antioxidant balance.

We found only two studies that investigate the relationship between OXS and manual therapy in the literature. According to these studies, manual treatment could reduce OXS by increasing the antioxidant enzyme capacity and decreasing the formation of products causing OXS. ${ }^{[12,13]}$ Highvelocity low amplitude manipulation (HVLAM) is an effective treatment for SIJD described by the fast and short pulse applied at the end of the passive range of movement. ${ }^{[11,14-17]}$ In our study, we aimed to investigate not only the effectiveness of HVLAM but also the relationship between SIJD, as a chronic pain and the oxidative system. We also intended to identify the factors associated with IMA, dynamic thiol/disulphide homeostasis, and thiol oxidation and the effects of treatments to reduce joint dysfunction in SIJD patients on OXS. Therefore, in this study, we aimed to investigate the effect of chiropractic manipulative treatment on SIJD and its relationship to OXS parameters.

\section{PATIENTS AND METHODS}

This cross-sectional, case-control study was conducted at Physical Medicine and Rehabilitation Department of Kirıkkale University Faculty of Medicine between February 2017 and September 2017. The study included 33 patients $(20$ males, 13 females; mean age $36.3 \pm 9.7$ years; range, 18 to 60 years) diagnosed with SI dysfunction in the outpatient clinic. Patients complained of pain in the SI joint region at least for the last three months. Patients' visual analog scale (VAS) score was at least 60 (median 81; range, 60 to 100). The required sample size was calculated by using a $\mathrm{G}^{*}$ power version 3.1.9.2, (Heinrich-Heine-Universität Düsseldorf, Düsseldorf, Germany). The power of the study was calculated as $92.40 \%$. This power is sufficient for a minimum of $80 \%$ power. The number of $\mathrm{n}$ was calculated as 25 , which would provide a standard effect size of 0.8 in the conditions of alpha 0.05 and beta 0.20 . Patients with chronic disorders (malignancy, thyroid dysfunction, obesity, hypertension, diabetes), neurological disease, infection, inflammatory rheumatologic disease, history of major lumbar and lower extremity surgery, pregnancy, spondylolisthesis and those using drugs (vitamin supplements, steroids, and nonsteroidal antiinflammatory, immunosuppressive) that can affect osteoporosis and OXS were excluded. In our study, palpation tests (Gillet test, standing and sitting flexion tests) and specific provocation tests (Gaenslen's and flexion abduction external rotation tests, and posterior shear or thigh thrust tests) were used. These tests had high clinical validity and reliability in patients with SI dysfunction. ${ }^{[18-21]}$ The control group consisted of age- and gender-matched 30 healthy individuals ( 20 males, 10 females; mean age $36.4 \pm 12.2$ years; range, 20 to 57 years) from the same center without being used in another study. They were without low back pain and systemic disease whose SI examination findings were normal. No payments were demanded from these persons or from their health insurance institutions. The study protocol was approved by the Kirikkale University Clinical Research Ethics Committee (01/10-03.01.2017). A written informed consent was obtained from each participant. The study was conducted in accordance with the principles of the Declaration of Helsinki.

At the baseline, a detailed history was obtained from each participant; musculoskeletal and SI joint pain-specific examinations were conducted and demographic information was recorded. Visual analog scale was used to evaluate pre- and post- 
treatment pain severity and intensity. Motion palpation and provocation tests were conducted at the beginning of the study and in the first month. Visual analog scale is a successful and commonly used method for assessing pain. In VAS, the severity of the pain is marked on a scale of $0-100 \mathrm{~mm} \cdot{ }^{[20-22]}$ Pre- and post-HVLAM evaluations (VAS, SIJD tests) were performed independently by the physician performing the manual treatment. In HVLAM, the patient was positioned on a treatment table in lateral recumbent position with the painful side up. The physiatrist stood against the patient. The physiatrist flexed the leg on the upper side until the lumbar spine was flexed and placed the foot in the popliteal fossa of the lower leg. Subsequently, the physiatrist seized the patient's lower shoulder and arm, and then performed hemi-trunk side bending and rotation till motion was felt at the SI joint. The patient's arms were put asround the physiatrist's arm. The patient was rolled toward the physiatrist while the setup was preserved. Thus, the spine was locked just above the SI joint, by creating a lever arm. Finally, the physiatrist pushed the SI joint posterior to anterior direction with high speed and low amplitude force (Figure 1). ${ }^{[13-16]}$ Usually, we used only one attempt for a single session that relieves the patient. Standards have not been defined in the manipulation therapy clearly and completely yet. Even so, according to analyses

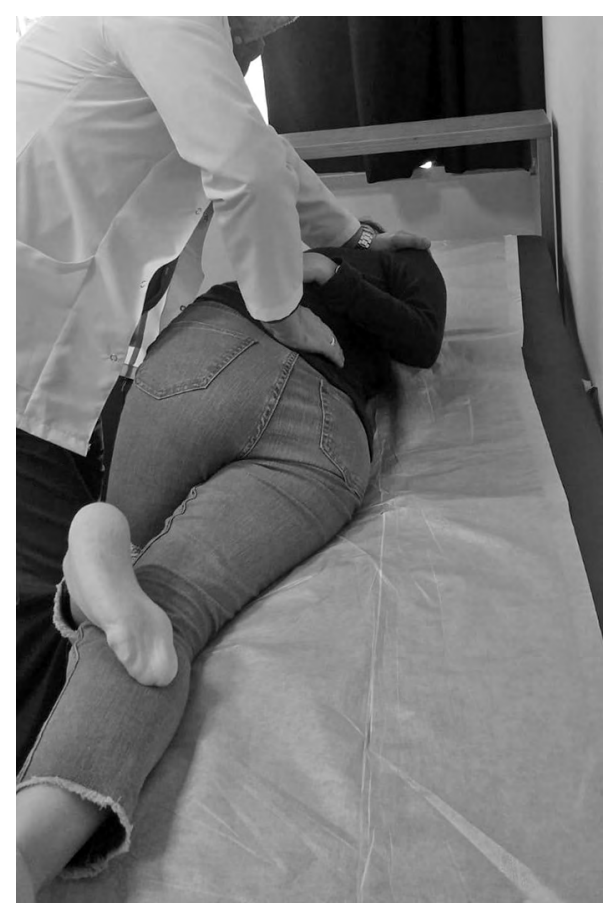

Figure 1. High velocity low amplitude manipulation in sacroiliac joint dysfunction. performed using various methods, manipulation applied to the SI region was a mean peak force of 210-240 $\mathrm{N}$ and a thrust phase duration of about $100 \mathrm{msn}$ for effective and successful HVLAM. In addition, these values indicate that HVLAM manual therapy does not pose a risk when administered with appropriate indications. Although there is no clear rule, it was reported that the application of HVLAM once a week for four weeks was effective in the relief of symptoms..$^{[15-19,23-31]}$

Clinical improvement was considered due to the applied treatment if the SI tests were negative and the VAS score was less than 60 . Patients who did not benefit from the treatment were excluded and the comparisons were repeated. A 5-mL blood sample before the treatment and a 5- $\mathrm{mL}$ after a week following the four weeks of manipulative therapy were collected from each patient with SIJD and control subject. All blood samples were taken after eight hours of fasting and centrifuged at $1500 \mathrm{rpm}$ for $10 \mathrm{~min}$ without waiting. Serum was separated and stored at $-80^{\circ} \mathrm{C}$ until analysis. All parameters were studied in separate serum samples. In this study, the thiol/disulphide balance and IMA concentration were evaluated by using a recently developed method. ${ }^{[7]}$

\section{Statistical analysis}

Statistical analysis was performed by using the SPSS version 16.0 software (SPSS Inc., Chicago, IL, USA). The confidence interval in the analysis was $95 \%$. The normality of parameters was tested by Shapiro-Wilk test $(\mathrm{p}<0.05$, the skewness-kurtosis tests and range between -1.96 and +1.96 assigned parametric as otherwise non-parametric). Parametric data were described as a mean \pm standard deviation, while non-parametric data were described as median (minimum-maximum). Parametric tests for dependence samples such as pre- and posttreatment were compared by using paired sample t-test. Parametric tests for independent samples such as patient and control groups were compared by using the two-independent samples t-test. Nonparametric dependent samples such as pre- and posttreatment VAS scores were analyzed by Wilcoxon test. Non-parametric independent samples such as body mass index (BMI) were compared by using the Mann-Whitney $U$ test. Categorical parameters such as gender were compared by chi-square, while pre- and post-treatment SIJD scores were compared by the Fisher's exact test. Categorical data were described as frequency and percentages. The correlations were assessed using Pearson's correlation 


\begin{tabular}{|c|c|c|c|c|c|c|c|c|c|c|c|}
\hline & \multirow{2}{*}{\multicolumn{5}{|c|}{ Control group $(n=30)$}} & \multirow{2}{*}{\multicolumn{5}{|c|}{$\begin{array}{l}\text { ent and control groups } \\
\text { Patient group }(n=33)\end{array}$}} & \multirow[b]{3}{*}{$p$} \\
\hline & & & & & & & & & & & \\
\hline & $\mathrm{n}$ & $\%$ & Mean \pm SD & Median & Min-Max & $\mathrm{n}$ & $\%$ & Mean \pm SD & Median & Min-Max & \\
\hline Age (year) & & & $36.4 \pm 12.2$ & & & & & $36.3 \pm 9.7$ & & & $0.97^{\star}$ \\
\hline BMI $\left(\mathrm{kg} / \mathrm{m}^{2}\right)$ & & & & 26.26 & $20.56-31.83$ & & & & 24.97 & $20.06-37.63$ & $0.88 \dagger$ \\
\hline Gender & & & & & & & & & & & $0.62 \ddagger$ \\
\hline Female & 10 & 33.3 & & & & 13 & 42.9 & & & & \\
\hline Male & 20 & 66.7 & & & & 20 & 57.1 & & & & \\
\hline $\begin{array}{l}\text { Disease duration } \\
\text { (month) } g\end{array}$ & & & & - & - & & & & 12 & $3-180$ & - \\
\hline VAS (median) & & & & - & - & & & & 81 & $60-100$ & - \\
\hline
\end{tabular}

coefficient for normal distribution samples and Spearman's correlation test for those not normally distributed. The way of the correlations was indicated as positive ( $r$ ) or negative (-r). The evaluation of the correlation of $\mathrm{r}$-value magnitudes was shown as very high $(0.90<\mathrm{r} \leq 1)$, high $(0.75<\mathrm{r} \leq 0.90)$, moderate $(0.50<\mathrm{r} \leq 0.75)$, low $(0.25<\mathrm{r} \leq 0.50)$, very low $(0<\mathrm{r} \leq 0.25)$. A $p$ value of $<0.05$ was considered statistically significant.

\section{RESULTS}

In the patient group, the median duration of low back pain was 12 months (range, 3 to 12 months). There was no difference between the patient and control groups in terms of age, gender, or BMI (Table 1). In the patient group, VAS scores were decreased significantly after HVLAM ( $\mathrm{p}=0.01)$ (Table 2).

The IMA $(\mu \mathrm{mol} / \mathrm{L})$ levels were higher in the patient group than the control group before the treatment $(0.9 \pm 0.1 \mu \mathrm{mol} / \mathrm{L}$ in pre-treatment patients; $0.8 \pm 0.1$ in control subjects; $\mathrm{p}=0.01$ ) (Table 2). The IMA levels were still higher in the patient group than the control group after the treatment $(0.9 \pm 0.1 \mu \mathrm{mol} / \mathrm{L}$ in posttreatment patients; $0.8 \pm 0.1 \mu \mathrm{mol} / \mathrm{L}$ in control subjects; $\mathrm{p}=0.01)$. There was no difference compared to pre and postreatment IMA levels $(\mathrm{p}=0.25)$.

The mean native thiol levels $(\mu \mathrm{mol} / \mathrm{L})$ were lower than the control group before treatment $(321.2 \pm 48.8$ $\mu \mathrm{mol} / \mathrm{L}$ in pre-treatment patients; $345.0 \pm 36.4 \mu \mathrm{mol} / \mathrm{L}$ in control subjects $p=0.03$ ). In addition, the mean native thiol level was even further lower, also persisted decrease after manipulation therapy $(\mathrm{p}=0.01)$. However, no significant difference was detected between the preand post-treatment of mean native thiol levels after manipulation therapy $(\mathrm{p}=0.18)$, which was $305.6 \pm 35.6$ $\mu \mathrm{mol} / \mathrm{L}$ in postreatmant patients and $321.2 \pm 48.8$ $(\mu \mathrm{mol} / \mathrm{L})$ in pretratment patients group.

Total thiol levels were significantly lower in the pre- and post-treatment groups compared to the control group $(360.6 \pm 46.9 \mu \mathrm{mol} / \mathrm{L}$ in pre-treatment patients; $343.6 \pm 38.2 \mu \mathrm{mol} / \mathrm{L}$ in post-treatment patients; $387.5 \pm 39.6 \mu \mathrm{mol} / \mathrm{L}$ in control subjects; $\mathrm{p}=0.01$ and $\mathrm{p}=0.01$, respectively). Total thiol levels did not differ significantly in pre- and post-treatment comparison $(\mathrm{p}=0.12)$ (Table 2).

As the mean levels of disulphide compared between all three groups with each other $(22.6 \pm 8.9 \mu \mathrm{mol} / \mathrm{L}$ in control subjects; $19.7 \pm 9.1 \mu \mathrm{mol} / \mathrm{L}$ in pretreatment patients; $18.9 \pm 8.5 \mu \mathrm{mol} / \mathrm{L}$ in post-treatment patients), we have found any difference that, control and pretreatment group $(\mathrm{p}=0.21)$, pre and pretreatment group $(p=0.78)$, control and pretreatment group $(\mathrm{p}=0.10)$.

The disulphide/native thiol proportion (SS/SH) found $6.6 \pm 2.6$ in control subjects; $6.4 \pm 3.5$ in pre-treatment and $6.3 \pm 2.9$ in post-treatment patients. When compared the disulphide/native thiol proportion ( $\mathrm{SS} / \mathrm{SH})$ between control- pretreatment, pre-postreatment, control-posttratment groups there was no significant difference in between groups ( $\mathrm{p}=0.82 ; \mathrm{p}=0.89, \mathrm{p}=0.66$ respectively) (Table 2 ).

Native thiol/total thiol proportion did not differ in any comparison between the control, pre- and posttreatment groups, similar to the SS/SH proportion $(89.1 \pm 0.0$ in control subjects; $89.0 \pm 5.4$ in pre-treatment and $89.1 \pm 4.7$ in post-treatment patients; $\mathrm{p}=0.88$; $\mathrm{p}=0.95 ; \mathrm{p}=0.95$, respectively) (Table 2 ). 
The VAS score was decreased significantly after treatment in the patient group. After the treatment, $78 \%$ of patients $(n=26)$ recovered from SIJD. After the manipulation theraphy, a significant decrease in VAS score was detected in those who had negative SIJD test. $(\mathrm{p}=0.01)$ (Table 2).

According to disease duration values, all patients had chronic pain and there was no correlation between the pain duration and the parameters before and after the treatment in the patient group. In the patient group, moderate negative correlations were determined between IMA and both native thiol $(\mathrm{r}=-0.52, \mathrm{p}=0.01)$ and total thiol $(\mathrm{r}=-0.51, \mathrm{p}=0.01)$.

\section{DISCUSSION}

In our study, IMA, as an oxidant source, ${ }^{[3,5-7]}$ was higher in the patient group than the control group before treatment that indicated the OXS. After treatment, IMA was still higher than the control group and there was no difference compared to pre-treatment levels. Thus OXS determined via IMA levels in the patients was more than the healthy control group. However, after our manipulative treatment, there was no significant difference in the patients. It was thought that OXS continued after treatment.

The disulphide bonds are formed by OXS. ${ }^{[3,5-7]}$ In terms of disulphide, there was no significant difference in levels among control subjects, and pre- and posttreatment patients. In other words, despite the decrease in substances of the antioxidant system, no increase was found in the disulphide oxidant. Therefore, the antioxidant system in patients with SIJD may decrease, while the oxidant system may not be influenced in preand post-manipulative treatment state.

The mean native thiol level, which is an antioxidant reservoir, was lower in the patient group than the control group before treatment. In addition, the mean native thiol level was even further lower after manipulative treatment. However, there was no difference between the pre- and post-treatment levels for native thiol levels. The results suggested that native thiol level was decreased in SIJD patients. Moreover, this condition persisted after manipulation therapy. In other words, this reserve was used in patients and continued to be used after manipulation.

In addition, total thiol levels were significantly lower in the pre-treatment patient group compared to the control group. In addition the mean total thiol levels were further decreased after the treatment. But

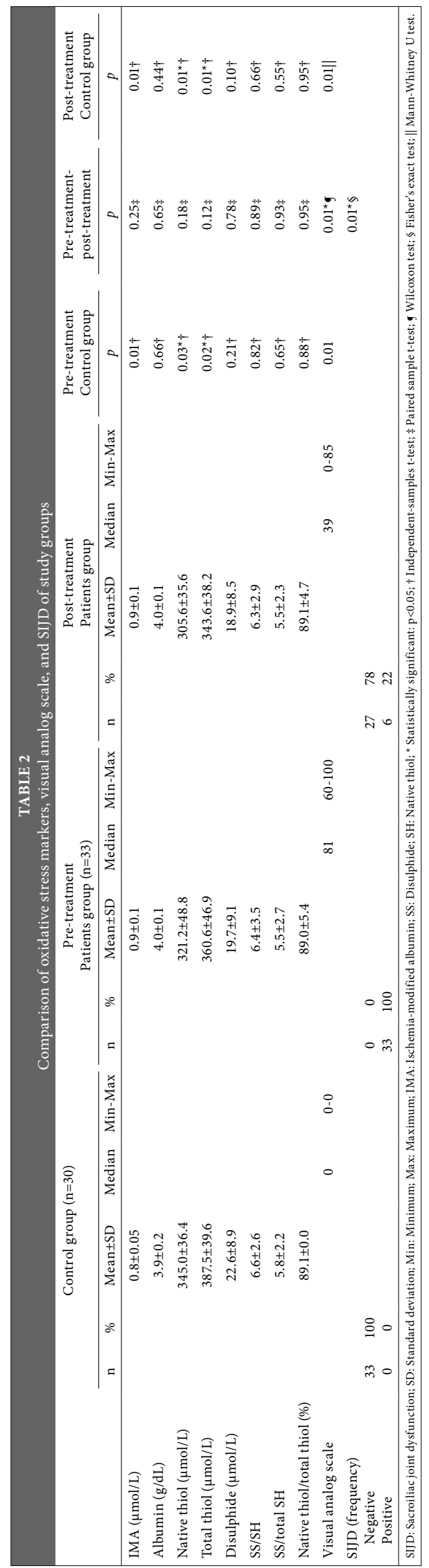


total thiol levels did not significantly differ when compared in pre and post-treatment groups Whereas total thiol value is not the sum of native thiol and disulfide bonds, but it shows all thiol levels. ${ }^{[3,5-7]}$ The decrease in the total thiol group would be due to the decrease in native thiol group.

The SS/SH proportion indicates oxidant/antioxidant status. This ratio did not differ between the groups in any comparison. On the other hand, native thiol/total thiol indicates the ratio of free thiol bonds to all thiol bonds. Similar to SS/SH proportion, this ratio did not differ between the groups in any comparison. These show that the antioxidant system may decrease, while OXS may increase. However, the oxidant system may not influence chronic pain such as SIJD. Considering the VAS value, it was determined that manipulation reduced pain while it did not eliminate OXS.

Thiol/disulphide balance shows that substances in the antioxidant system (e.g. native and total thiol) decrease in a patient with chronic pain. Dogru et al. ${ }^{[6]}$ investigated the thiol/disulphide balance in 69 patients with AS and compared the results with 60 healthy controls. They found that the total thiol levels in the patient group were significantly lower than the control group ${ }^{[6]}$ In this study, their patient group was divided into two groups as active and inactive, according to the Bath Ankylosing Spondylitis Disease Activity Index and VAS scores and the authors found that the levels of both native and total thiols were statistically lower in the active group. In addition, La Rubia et al. ${ }^{[7]}$ investigated OXS (lipid and protein peroxidation and oxidative DNA damage) in 45 females with FM and 25 healthy controls. In their study, the authors analyzed the total antioxidant capacity and antioxidant enzyme activity and its compounds. They found that oxidative damage and total antioxidant capacity and enzyme activities such as superoxide dismutase, glutathione peroxidase, and catalase decreased in the group with FM. In addition, Toker et al. ${ }^{[8]}$ in 59 FM patients found that serum IMA values were significantly higher than the healthy control group. Chronic pain affects the oxidative system and weakens the antioxidant defense system by causing oxidative damage. Kohlberg et al. $^{[12,13]}$ reported that manipulative treatment could reduce pain according to studies evaluating pain severity with VAS scale. In their studies, manipulative treatment was applied twice a week for five weeks in 23 patients with chronic neck and back pain. They measured before and after levels of superoxide dismutase, catalase, glutathione peroxidase, nitric oxide metabolites and lipid hydroperoxides levels which are antioxidant enzymes. The superoxide dismutase levels were found to have increased compared to pre-treatment. However, catalase and nitric oxide metabolites and lipid hydroperoxide levels did not change after treatment. ${ }^{[12,13]}$

In this study, no statistically significant difference was detected between oxidative and antioxidative markers in patients before and after HVLAM. These findings suggest that in the short term, manual therapy may not have any significant effect on the oxidantantioxidant balance. As known, nociceptors involved in pain transmission to the central nervous system modulate reactive oxygen species and increase their production. Pain also increases reactive oxygen and nitrogen products by neuronal stimulation, and even treatment with antioxidant compounds displays antinociceptive effects. ${ }^{[4,28,29]}$ Further research is needed to determine the harmful and beneficial aspects of the decrease in substances of the antioxidant system in SIJD patients. According to some authors, manual therapy was the best treatment in SIJD ${ }^{[30]}$ However, according to the literature, the efficiency of manipulative treatment in SIJD was found to be at moderate to a weak level. Researchers have emphasized that results are open to debate and that there is a need for more controlled and better-designed studies. ${ }^{[31]}$ Our study is important in that the manual treatment in short term did not increase the substances of the antioxidant system. This may be a useful defense mechanism for the organism with the condition that it does not lead to OXS. ${ }^{[1-8]}$ Further studies should explain whether the effect of this change on the organism is positive or negative. Ischemia-modified albumin, as an oxidative marker, has a negative correlation with antioxidant markers of total and native thiol, suggesting that these markers may be used to follow OXS in SIJD.

This study has some limitations. First, the low number of patients may have affected this results. Second, the change in long-term oxidative parameters was not evaluated. Third, although local anesthetic injection in the SI joint is the gold standard method to evaluate SI dysfunction, the injection was not included in the study design because the majority of patients did not accept it.

In conclusion, serum total thiol, native thiol, disulphide, and IMA may be used to measure the oxidative and antioxidative systems in SIJD patients with chronic pain due to mild musculoskeletal problems. High-velocity low amplitude manipulation techniques can be beneficial for chronic pain in patients with SIJD. In addition, HVLAM is not traumatic or harmful to the organism. However, HVLAM 
has not eliminated the OXS. Currently, the serum thiol/disulphide balance and IMA measurements are cost-effective and simple oxidative system parameters in musculoskeletal diseases such as SIJD.

\section{Declaration of conflicting interests}

The authors declared no conflicts of interest with respect to the authorship and/or publication of this article.

\section{Funding}

The authors received no financial support for the research and/or authorship of this article.

\section{REFERENCES}

1. Marchev AS, Dimitrova PA, Burns AJ, Kostov RV, DinkovaKostova AT, Georgiev MI. Oxidative stress and chronic inflammation in osteoarthritis: can NRF2 counteract these partners in crime? Ann N Y Acad Sci 2017;1401:114-35.

2. Sun CL, Wei J, Bi LQ. Rutin Attenuates Oxidative Stress and Proinflammatory Cytokine Level in Adjuvant Induced Rheumatoid Arthritis via Inhibition of NF-kB. Pharmacology 2017;100:40-9.

3. Philippe C, Wittrant Y. Oxidative stress in musculoskeletal disorders-bone disease. In: Laher I, editor. System Biology of Free Radicals and Antioxidants. Berlin Heidelberg; 2014. p. 2951-9.

4. Cheng YY, Kao CL, Ma HI, Hung CH, Wang CT, Liu $\mathrm{DH}$, et al. SIRT1-related inhibition of pro-inflammatory responses and oxidative stress are involved in the mechanism of nonspecific low back pain relief after exercise through modulation of Toll-like receptor 4 . J Biochem 2015;158:299-308.

5. Erel O, Neselioglu S. A novel and automated assay for thiol/disulphide homeostasis. Clin Biochem 2014;47:326-32.

6. Dogru A, Balkarli A, Cetin GY, Neselioglu S, Erel O, Tunc SE, et al. Thiol/disulfide homeostasis in patients with ankylosing spondylitis. Bosn J Basic Med Sci 2016;16:187-92.

7. La Rubia M, Rus A, Molina F, Del Moral ML. Is fibromyalgia-related oxidative stress implicated in the decline of physical and mental health status? Clin Exp Rheumatol 2013;31:S121-7.

8. Toker A, Kucuksen S, Kucuk A, Cicekler H. Serum ischemiamodified albumin and malondialdehyde levels and superoxide dismutase activity in patients with fibromyalgia. Clin Lab 2014;60:1609-15.

9. Türkön H, Gökmen F, Çakir DÜ, Sehitoğlu MH, Reşorlu H, Döner D, et al. Increased Levels of Serum Ischemia Modified Albumin in Patients with Ankylosing Spondylitis. Clin Lab 2016;62:645-9.

10. Leitemperguer MR, Tatsch E, Kober H, De Carvalho JA, Moresco RN, Da Silva JE. Assessment of ischemia modified albumin levels in patients with rheumatoid arthritis. Clin Lab 2014;60:1065-70.

11. Farazdaghi MR, Motealleh A, Abtahi F, Panjan A, Šarabon N, Ghaffarinejad F. Effect of sacroiliac manipulation on postural sway in quiet standing: a randomized controlled trial. Braz J Phys Ther 2018;22:120-6.
12. Kolberg C, Horst A, Moraes MS, Duarte FC, Riffel AP, Scheid T, et al. Peripheral oxidative stress blood markers in patients with chronic back or neck pain treated with high-velocity, low-amplitude manipulation. J Manipulative Physiol Ther 2015;38:119-29.

13. Kolberg C, Horst A, Moraes MS, Kolberg A, Belló-Klein A, Partata WA. Effect of high-velocity, low-amplitude treatment on superoxide dismutase and glutathione peroxidase activities in erythrocytes from men with neck pain. J Manipulative Physiol Ther 2012;35:295300.

14. Kamali F, Shokri E. The effect of two manipulative therapy techniques and their outcome in patients with sacroiliac joint syndrome. J Bodyw Mov Ther 2012;16:29-35.

15. Cerqueira MS, Sales RM, Pinto CTP, Moreno BGD, Moura Filho AG. High-velocity low amplitude manipulation (thrust) and athletic performance: a systematic review. Fisioter Mov 2017;30:413-22.

16. Clar C, Tsertsvadze A, Court R, Hundt GL, Clarke A, Sutcliffe P. Clinical effectiveness of manual therapy for the management of musculoskeletal and non-musculoskeletal conditions: systematic review and update of UK evidence report. Chiropr Man Therap 2014;22:12.

17. Isaacs ER, Bookhout MR. Treatment of dysfunctions of the pelvis. In: Isaacs ER, Bookhout MR, editors. Bourdillon's Spinal Manipulation. 6th ed. Boston: Butterworth Heinemann; 2002. p. 125-56.

18. Laslett M, Aprill CN, McDonald B, Young SB. Diagnosis of sacroiliac joint pain: validity of individual provocation tests and composites of tests. Man Ther 2005;10:207-18.

19. Szadek KM, van der Wurff P, van Tulder MW, Zuurmond WW, Perez RS. Diagnostic validity of criteria for sacroiliac joint pain: a systematic review. J Pain 2009;10:354-68.

20. Hjermstad MJ, Fayers PM, Haugen DF, Caraceni A, Hanks GW, Loge JH, et al. Studies comparing Numerical Rating Scales, Verbal Rating Scales, and Visual Analogue Scales for assessment of pain intensity in adults: a systematic literature review. J Pain Symptom Manage 2011;41:1073-93.

21. Kersten $\mathrm{P}$, Küçükdeveci AA, Tennant A. The use of the Visual Analogue Scale (VAS) in rehabilitation outcomes. J Rehabil Med 2012;44:609-10.

22. Foley BS, Buschbacher RM. Sacroiliac joint pain: anatomy, biomechanics, diagnosis, and treatment. Am J Phys Med Rehabil 2006;85:997-1006.

23. Nielsen SM, Tarp S, Christensen R, Bliddal H, Klokker L, Henriksen $\mathrm{M}$. The risk associated with spinal manipulation: an overview of reviews. Syst Rev 2017;6:64.

24. Evans DW. Mechanisms and effects of spinal high-velocity, low-amplitude thrust manipulation: previous theories. J Manipulative Physiol Ther 2002;25:251-62.

25. Downie AS, Vemulpad S, Bull PW. Quantifying the high-velocity, low-amplitude spinal manipulative thrust: a systematic review. J Manipulative Physiol Ther 2010;33:542-53.

26. Bronfort G, Haas M, Evans RL, Bouter LM. Efficacy of spinal manipulation and mobilization for low back pain and neck pain: a systematic review and best evidence synthesis. Spine J 2004;4:335-56. 
27. Gudavalli MR, Cambron JA, McGregor M, Jedlicka J, Keenum M, Ghanayem AJ, et al. A randomized clinical trial and subgroup analysis to compare flexion-distraction with active exercise for chronic low back pain. Eur Spine J 2006;15:1070-82.

28. Scheid T, Bosco LD, Guedes RP, Pavanato MA, BellóKlein A, Partata WA. Sciatic nerve transection modulates oxidative parameters in spinal and supraspinal regions. Neurochem Res 2013;38:935-42.

29. Hjornevik T, Jacobsen LM, Qu H, Bjaalie JG, Gjerstad J, Willoch F. Metabolic plasticity in the supraspinal pain modulating circuitry after noxious stimulus-induced spinal cord LTP. Pain 2008;140:456-64.

30. Sharma A, Sharma S, Steiner LA, Brudvig TJ. Identification and effectiveness of physical therapy interventions for sacroiliac joint dysfunction in pregnant and nonpregnant adults: a systematic review. Journal of Women's Health Physical Therapy 2014;38:110-7.

31. Al-Subahi M, Alayat M, Alshehri MA, Helal O, Alhasan $\mathrm{H}$, Alalawi A, et al. The effectiveness of physiotherapy interventions for sacroiliac joint dysfunction: a systematic review. J Phys Ther Sci 2017;29:1689-94. 